\title{
Fuel Cost Optimization of an Islanded Microgrid Considering Environmental Impact
}

\author{
Vinod M. Raj and Saurabh Chanana
}

\begin{abstract}
This paper shows how the total fuel cost generated by the distributed generating units of a microgrid is minimized by the application of unit commitment problem. The microgrid taken for case study consists of a wind power unit, a photovoltaic unit, a microturbine, a diesel generator and a battery bank. The unit commitment problem is solved using priority listing method. The priority is assigned on the basis of emissions released by each unit. The 24 hour forecasted power output of the renewable energy sources is subtracted from the given 24 hour load requirement and is not considered in the unit commitment problem. The fuel cost coefficients and generating constraints of the conventional units and the battery bank specifications are taken as data for solving the unit commitment problem. Minimum up time, minimum down time and startup costs of the conventional units are considered in this case study. Ramp rate constraints and reserve capacity constraints are ignored and shut down cost is taken as zero. Operating costs of the renewable units are taken as zero and the cost of energy delivered during battery discharge is considered as profit. The economic dispatch problem is solved using Quadratic Programming approach. Losses in the system are ignored while solving the economic dispatch problem. From the results obtained we can conclude that even if the unit commitment schedule generated may not be most economic, it still ensures an environment friendly operation by the microgrid.
\end{abstract}

Index Terms-Microgrid, priority listing, quadratic programming, unit commitment problem.

\section{INTRODUCTION}

The unit commitment problem is generally applied in the field of power systems to find out the optimum generating schedule of the generating units to satisfy the load demand. The optimal scheduling of power is to be done in a way such that all constraints of the generating systems and the network are met. The unit commitment time period generally varies from one day to one week. It requires solving the unit commitment problem for each hour. Various optimization algorithms are applied in this problem to achieve the optimum power generation. The optimal fuel cost generated is calculated with the help of the power output obtained by the application of the optimization techniques.

The technique used for solving the unit commitment problem mainly depends on the data that is available and the constraints in the problem. Sometimes the fuel cost function is quadratic or convex in nature and hence in such cases Priority Listing method or Lagrange method can be applied to solve the problem. However if the problem is non-convex, we need to use dynamic programming or other meta-heuristic

Manuscript received November 30, 2014; revised April 23, 2015.

The authors are with the Electrical Engineering Department, National Institute of Technology Kurukshetra, India (e-mail: vinodmraj1@ gmail.com, s_chanana@rediffmail.com). approaches to solve the problem.

In recent times, the traditional power system network is gradually transforming from a centralized grid to a cluster of localized distributed generation networks. The main reason for these changes is the restructuring of the power system network resulting due to various revisions of the electricity act. These localized distributed generating networks are termed as microgrids. They have the ability to work in synchronism with the main grid as well as in isolation from the main grid. These microgrids are generally comprised of low power combined heat and power plants, renewable energy sources and energy storage devices. The overall power and voltage level of a microgrid is quite lower than that of the main grid. The main advantages of a microgrid are lower transmission losses, lesser carbon emissions, more reliability since it can operate in islanded mode and is hence free from the interruptions caused due to power outages in the main grid. However, some complexities arise when solving the unit commitment problem for a microgrid. The fuel cost objective function of a microgrid is a mixture of quadratic, linear and undefined entities because of the presence of renewable energy sources and energy storage devices. It becomes even more difficult when we schedule the buying and selling of power from the main grid when the microgrid is working in interconnected mode.

In the literature survey, [1] has solved the unit commitment problem for a microgrid in islanded mode as well as interconnected mode using Priority Listing method and Genetic Algorithm. Reference [2] has done sizing and the optimal allocation of distributed generation and has also performed economic dispatch calculations considering emissions of each unit. In [3], optimization of fuel cost of an interconnected microgrid by performing a sensitivity analysis on the pricing structure of energy transfer from the main grid has been done. There is also a market based survey on the regulatory bodies of the electricity market. Reference [4] has applied the Mixed Integer Linear Programming approach to optimize the microgrid as well as schedule the buying and selling of power from the main grid. It has also imposed a linear diversity constraint upon the system. In [5], electric as well as thermal demand has been considered in the optimization problem and has also included energy storage units in the microgrid. A review on existing microgrid systems all over the world has been presented in [6]. Authors have highlighted some important parts of technology used in some of the systems which are important from point of view of research and further enhancement of the operation of microgrids. Reference [7] has determined the electricity transfer price between the microgrid and the main grid by using Game Theory Nash bargaining solution approach. The remaining part of optimizing the microgrid operation is done 
through Mixed Integer programming. In [8], the unit commitment problem in the microgrid has been solved using Genetic Algorithm approach. A Mixed Integer Non Linear Programming approach has been used in [9] to solve economic dispatch problem in a microgrid consisting of Combined Heat and Power plants. Reference [10] has solved the economic dispatch for a microgrid using Particle Swarm Optimization approach and has studied the impact of battery charging on the optimization process.

In this paper, Priority Listing method is applied to solve the unit commitment problem in the given distributed energy system. The specifications of the generating units are described in Table I. Normally priority is assigned on the basis of the cost of each generator. But in this case the priority is assigned on the basis of the emission released by the generating unit. That is, first priority is given to the unit which causes least pollution. The economic dispatch calculations are carried out using Quadratic Programming approach. The content of the paper is as follows: Section II talks about the objective function that has to be minimized and the constraints which have to be met. Section III gives the generator data namely the cost coefficients and the generating limits. Section IV shows the results obtained through priority listing. Section V shows the conclusion obtained from the above results and the future work to be done in the area of unit commitment.

\section{MATHEMATICAL MODEL}

\section{A. Nomenclature}

\section{- Acronyms}

MT - Microturbine

DG - Diesel Generator

PV - Photo Voltaic unit

WT - Wind Turbine

BB - Battery Bank

SOC - State of Charge

- Indices

$i$ - Unit number

$t$ - Time interval

- Parameters

$T$ - Total number of hours

$N$-Total number of units

$\operatorname{Pmax}_{i}$ - Maximum generation capacity of unit $i$

$\operatorname{Pmin}_{i}$ - Minimum generation capacity of unit $i$

$S O C_{\text {min }}$ - Minimum state of Charge of BB

$S O C_{\max }-$ Maximum state of Charge of $\mathrm{BB}$

$\mathrm{Tup}_{i}-$ Minimum up time of unit $i$

Tdown $i$ - Minimum down time of unit $i$

$S C_{i}-$ Start up cost of unit $i$

$a_{i}, b_{i}$ and $c_{i}-$ Coefficients of the quadratic cost function of unit $i$

$P_{D t}-$ Load demand at hour $t$

$F_{t}-$ Fuel Cost for hour $t$

$d_{t}-$ Battery incremental cost for hour $t$

$F_{B t}-$ Battery charging cost

$P_{B t}-$ Battery charging power

- Variables

$P_{i t}-$ Power of unit $i$ in hour $t$
$C_{i}\left(P_{i t}\right)-$ Cost function of unit $i$

$F_{T}-$ Total Fuel Cost

$S O C_{t}$ - State of charge of battery at hour $t$

- Variables used in Quadratic Programming Algorithm

$k$ - Iteration index

$i-$ Variable index

$N$ - Total number of variables

$S_{k}$-Matrix representing constraint expressions

$p-$ a vector of constants

$c-$ a vector of size $\mathrm{N}$ having constants

$h-$ a vector of size $\mathrm{NxN}$ having constants

$x-$ a vector of size $\mathrm{N}$ having variables

$F(x)$-The objective function for quadratic programming which has to be minimized

$d_{k}-$ Search direction matrix. Basically it is the set of initial values of $x$

$Z_{k}$ - Matrix orthogonal to $S_{k}$

$u b_{i}$-upper bound of variable $x_{i}$

$l b_{i}$ - lower bound of variable $x_{i}$

$A, b-$ Vectors satisfying equality constraint expressions $\alpha$ - convergence value

\section{B. Objective Function}

The cost function which has to be minimized consists of the operating cost and startup cost of the individual units and operational cost of battery which is as follows:

$$
F_{T}=\min _{P_{i t}} \sum_{t=1}^{T} \sum_{i=1}^{N}\left(\left(C_{i}\left(P_{i t}\right)+S C_{i t}\right)+F_{B t}\right)
$$

The individual cost functions are given as follows:

$$
\begin{gathered}
C_{i}\left(P_{i t}\right)=a_{i}+b_{i} P_{i t}+c_{i}\left(P_{i t}\right)^{2} \\
F_{B t}=d_{t} P_{B t}
\end{gathered}
$$

The incremental cost of battery is dependent on the state of charge as given below,

$$
d_{t}=\frac{S O C_{t-1} d_{t-1}+\left(S O C_{t}-S O C_{t-1}\right) F_{t-1}}{S O C_{t}}
$$

\section{Constraints}

The following constraints have been considered in the economic dispatch calculations

- Power Balance:

$$
\sum_{i=1}^{N} P_{i t}=P_{D t}
$$

- Generation limits:

$$
\operatorname{Pmin}_{i} \leq P_{i t} \leq \operatorname{Pmax}_{i}
$$

The constraints which are used in unit commitment formulation are as follows:

- Minimum up time $\left(\right.$ Tup $\left._{i}\right)$

Minimum up time is the minimum number of hours the unit is required to be in on state after it has been turned on.

- Minimum down time $\left(\right.$ Tdown $\left._{i}\right)$

Minimum down time is the minimum number of hours the unit is required to be in off state once it has been turned off. 


\section{Quadratic Programming Approach for Economic Dispatch}

Quadratic programming approach is applied in those areas where the objective function to be minimized is quadratic in nature [11]. In this case the cost function to be minimized given in equation (1) is in quadratic form. This equation is converted into the following form.

$$
F(x)=\min \left(c^{T} x+\frac{1}{2} x^{T} h x\right)
$$

In the above equation, variable set $x$ represents $P_{i t}$ and the above function is subject to the equality constraint given by $A x=b$ which is simply a matrix representation of equation (5). The upper and lower bounds of $x, u b_{i}$ and $l b_{i}$ are sets of $\operatorname{Pmax}_{i}$ and $\operatorname{Pmin}_{i}$ respectively as shown in (6). This technique is used to find the economic dispatch solution for each hour.

The above problem is solved using inbuilt quadratic programming toolbox of MATLAB. This optimization problem falls under medium scale optimization category according to MATLAB [12]. The algorithm for this technique is as follows:

- Construct the objective function to be minimized according to equation (7). Initialize iteration $k=1$, Construct matrix $S_{k}$ which is an estimate of the constraints that are satisfied. Find $Z_{k}$ such that $S_{k} Z_{k}=0$.

- Select search direction $d_{k}$ such that $d_{k}=Z_{k} p$. Vector $p$ is arbitrarily selected.

- The objective function can be modified as follows:

$$
\begin{gathered}
q(p)=\frac{1}{2} p^{T} Z_{k}^{T} h Z_{k} p+c^{T} Z_{k} p \\
\nabla q(p)=Z_{k}^{T} h Z_{k} p+Z_{k}^{T} c
\end{gathered}
$$

For minimum value of $q(p), \nabla q(p)=0$. Hence we get a set of linear equations that can be solved which are as follows:

$$
Z_{k}^{T} h Z_{k} p=-Z_{k}^{T} c
$$

- Now find value of $p$ and determine $d_{k}$ as shown in step 2 and update the value of $Z_{k}$ and $S_{k} €$

- Now find out the convergence value given by

$$
\alpha=\min \left\{\frac{-\left(A_{i} x_{k}-b_{i}\right)}{A_{i} d_{k}}\right\}
$$

- If value of $\alpha$ is less than the tolerance limit set, then assign $x=d_{k}$ and terminate the procedure. If not then update value of $x$ in the following way and repeat the steps from equation (10) onwards.

$$
x_{k+1}=x_{k}+\alpha d_{k}
$$

The flow chart for this algorithm is shown in Fig. 1.

\section{E. Priority Listing Method}

In this method the sequence for switching on and off of subsequent units is done by first calculating the full load average cost of each unit. Highest priority is given to the unit which has the lowest full load average production cost [13]. Hence for a given range of load demand we get a combination of the units required to be in on state. The unit having highest priority is switched on first and the unit having lowest priority is switched off first. This is the procedure that is followed for conventional units.

However in this case study, priority is assigned on the basis of emission generated by each unit. Therefore, first priority is given to the renewable energy sources, then the microturbine and finally the Diesel Generator. The battery bank is switched on and off on basis of the load demand. It is kept in charging mode during the off peak hours and in discharging mode during peak hours.

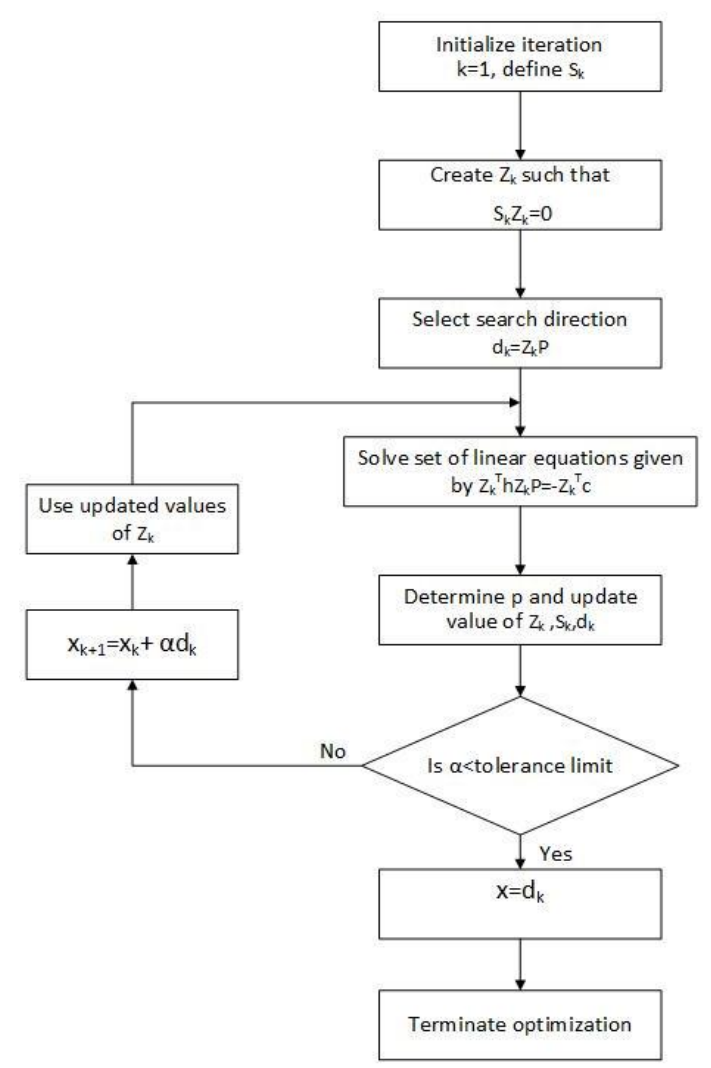

Fig. 1. Medium scale quadratic programming approach.

\section{PROBLEM DATA}

In the unit commitment problem, a five unit system is shown out of which two are renewable energy sources and one is a battery bank unit [1]. These units are committed to serve a 24 hour load pattern given in Fig. 2. Table I shows the unit data namely cost coefficients and generation constraints.

TABLE I: UNIT SPECIFICATIONS

\begin{tabular}{llllll}
\hline \hline & MT & DG & WT & PV & BB \\
\hline Pmax $(\mathrm{kW})$ & 50 & 150 & 80 & 40 & 120 \\
Pmin $(\mathrm{kW})$ & 5 & 15 & 0 & 0 & 0 \\
$\mathrm{a}(€ / \mathrm{h})$ & 2.62 & 0.6 & - & - & - \\
$\mathrm{b}(€ / \mathrm{kWh})$ & 0.15 & 0.05 & - & - & - \\
$\mathrm{c}\left(€ / \mathrm{kW}^{2} \mathrm{~h}\right)$ & 0.15 & 0.02 & - & - & - \\
SC $(€)$ & 2 & 5 & 0 & 0 & 0 \\
Tup $(\mathrm{h})$ & 1 & 3 & 0 & 0 & 0 \\
Tdown $(\mathrm{h})$ & 1 & 3 & 0 & 0 & 0 \\
Priority & 2 & 3 & 1 & 1 & - \\
Storage & - & - & - & - & 350 \\
Capacity $(\mathrm{kWh})$ & & & & & \\
SOC $_{\min }(\mathrm{kWh})$ & - & - & - & - & 70 \\
SOC $_{\max }(\mathrm{kWh})$ & - & - & - & - & 280 \\
\hline \hline
\end{tabular}




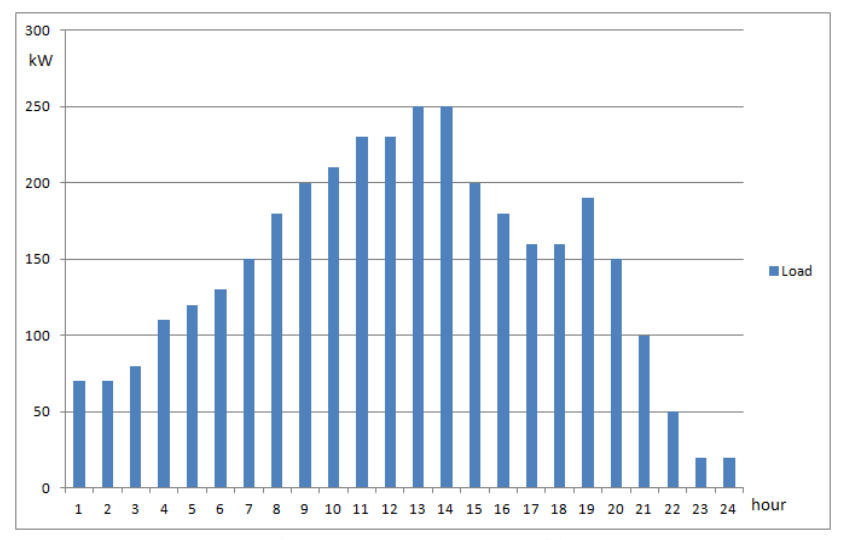

Fig. 2. Load demand profile.

The forecasted power output of the wind power unit and the Photo Voltaic unit is given in Fig. 3.

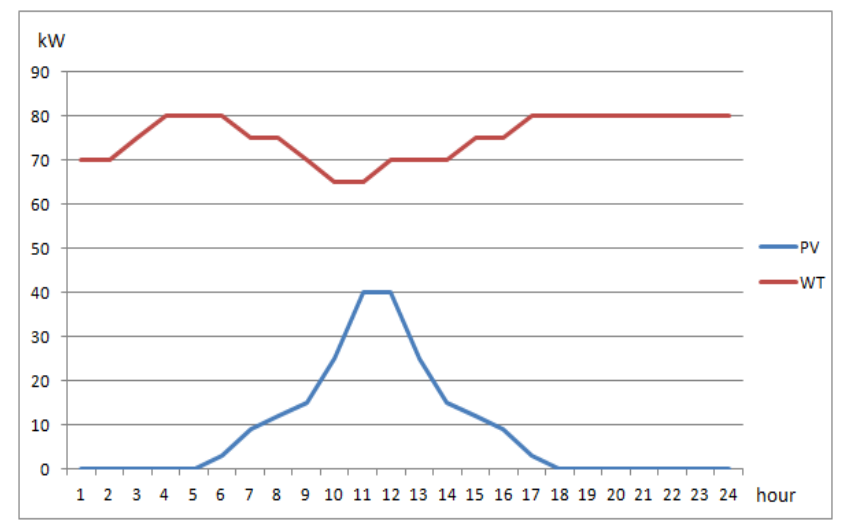

Fig. 3. Predicted output of solar and wind power unit.

A unit commitment schedule is to be generated for the given system such that the load demand for each hour is satisfied with optimum generation of active units leading to economic operation.

\section{RESULTS}

The unit commitment schedule is generated by running the code based on Priority Listing method on MATLAB for the given unit commitment problem. The output of each unit is shown in Fig. 4. The net operating cost obtained is described in Table II.

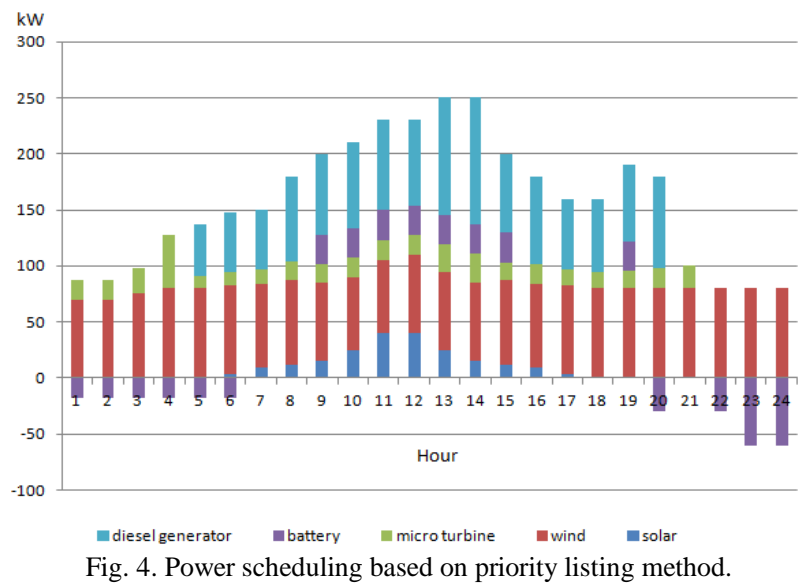

The battery output for some hours is shown negative. This represents the charging state of the battery bank. The power output of the wind and solar units are fixed and do not play any role in the optimization procedure. Their running costs are taken as zero.

TABLE II: GENERATION COST USING PRIORITY LISTING

\begin{tabular}{|c|c|c|c|}
\hline Hour & $\begin{array}{c}\text { Running } \\
\text { Cost }(€)\end{array}$ & $\begin{array}{c}\text { Startup } \\
\operatorname{cost}(€)\end{array}$ & $\begin{array}{c}\text { Battery Charging } \\
\operatorname{cost}(€)\end{array}$ \\
\hline 1 & 51 & - & 158 \\
\hline 2 & 51 & - & 149 \\
\hline 3 & 82 & - & 141 \\
\hline 4 & 348 & - & 136 \\
\hline 5 & 68 & 5 & 135 \\
\hline 6 & 84 & - & 135 \\
\hline 7 & 88 & - & 0 \\
\hline 8 & 169 & - & 0 \\
\hline 9 & 155 & - & -191 \\
\hline 10 & 172 & - & -178 \\
\hline 11 & 190 & - & -160 \\
\hline 12 & 172 & - & -134 \\
\hline 13 & 318 & - & -102 \\
\hline 14 & 368 & - & -32 \\
\hline 15 & 148 & - & 84 \\
\hline 16 & 180 & - & 0 \\
\hline 17 & 118 & - & 0 \\
\hline 18 & 127 & - & 0 \\
\hline 19 & 138 & - & 218 \\
\hline 20 & 195 & - & -77 \\
\hline 21 & 66 & - & 0 \\
\hline 22 & 0 & - & -36 \\
\hline 23 & 0 & - & -50 \\
\hline 24 & 0 & - & -38 \\
\hline Total & 3288 & 5 & 156 \\
\hline Net $\operatorname{Cost}(€)$ & 3449 & & \\
\hline
\end{tabular}

The battery charging costs which are in negative indicate that either the battery bank is in discharge mode or the value of $d_{t}$ (given in equation (4)) is negative.

\section{CONCLUSION AND FUTURE WORK}

The above results are obtained by assigning the priority on basis of emission released by each generator. Hence the overall fuel cost obtained may not be most economic. But the unit commitment schedule generated is intended to keep emissions at a lower value. This ensures a cleaner generation by the microgrid. Future work will involve implementation of unit commitment problem for a microgrid interconnected with the main grid and scheduling of buying and selling of power. Optimization techniques different from those mentioned in this paper will be used for solving the unit commitment and economic dispatch problems.

\section{REFERENCES}

[1] J. P. Fossati, "Unit commitment and economic dispatch in micro grids," Memoria de Trabajos de Difusión Científica y Técnica, no. 10, pp. 83-96, September 2012.

[2] A. K. Basu, A. Bhattacharya, S. Chowdhury, S. P. Chowdhury, "Planned scheduling for economic power sharing in a CHP-based micro-grid," IEEE Transactions on Power Systems, vol. 27, no. 1, pp 30-38, February 2012.

[3] A. D. Hawkes and M. A. Leach, "Modelling high level system design and unit commitment for a microgrid," Applied Energy, vol. 86, pp. 1253-1265, October 2008

[4] P. K. Naraharisetti, I. A. Karimi, A. Anand, and D.-Y. Lee, "A linear diversity constraint - Application to scheduling in microgrids," Energy, vol. 36, pp. 4235-4243, May 2011. 
[5] J. Aghaei and M.-I. Alizadeh, "Multi-objective self-scheduling of CHP (combined heat and power)-based microgrids considering demand response programs and ESSs (energy storage systems)," Energy, vol. 55, pp. 1044-1054, May 2013.

[6] N. W. A. Lidula and A. D. Rajapakse, "Microgrids research: A review of experimental microgrids and test systems," Renewable and Sustainable Energy Reviews, vol. 15, pp. 186-202, September 2010.

[7] D. Zhang, N. J. Samsatli, A. D. Hawkes, D. J. L. Brett, N. Shah, and L. G. Papageorgiou, "Fair electricity transfer price and unit capacity selection for microgrids," Energy Economics, vol. 36, pp. 581-593, November 2012

[8] H. Z. Liang and H. B. Gooi, "Unit commitment in microgrids by improved genetic algorithm," in Proc. IPEC 2010, pp. 842-847.

[9] J. S. Kim and T. F. Edgar, "Optimal scheduling of combined heat and power plants using mixed-integer nonlinear programming," Energy, vol. 77, pp. 675-690, October 2014

[10] H. Wu, X. Liu, and M. Ding, "Dynamic economic dispatch of a microgrid: Mathematical models and solution algorithm," Electrical Power and Energy Systems, vol. 63, pp. 336-346, July 2014.

[11] G. F. Reid and L. Hasdorff, "Economic dispatch using quadratic programming," in Proc. IEEE PES Winter Meeting, New York, February 1973, pp. 2015-2023.

[12] MATLAB 2010 Help Manual, the MathWorks Inc., 2010.

[13] A. J. Wood and B. F. Wollenberg, Power Generation, Operation and Control, $2^{\text {nd }}$ ed., John Wiley and Sons Inc., New York, 1996, ch. 5.

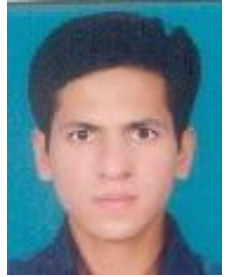

formulation.

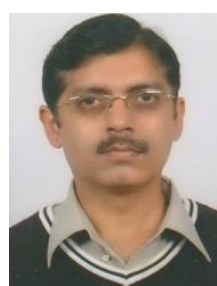

Vinod M. Raj received his B.Tech degree from Nirm Institute of Technology, India in 2011. He has worked at ERDA (Electrical Research and Development Association), India. $\mathrm{He}$ is currently pursuing his M.Tech degree at the Department of Electrical Engineering, National Institute of Technology Kurukshetra, India. His area of interest is the application of optimization techniques in unit commitment and economic load dispatch problem of power system restructuring, real-time pricing, demand response and grid integration of renewable energy. He is member of IEEE Power and Energy Society, Control System Society and Industry Application Society. He is a life member of Indian Society of Technical Education over fifteen years. His research interests are in the area

Saurabh Chanana received his B.Tech., M.Tech. and $\mathrm{Ph} . \mathrm{D}$. degrees from National Institute of Technology Kurukshetra, India in 1996, 2002, and 2011 respectively. He has worked with DLF Universal Ltd presently as an associate professor at the Department of Electrical Engineering, National Institute of Technology Kurukshetra. $\mathrm{He}$ has been teaching subjects in the area of power system technology for rid 